\title{
Instantons and Representations of an Associative Algebra
}

\author{
Yoshitake Hashimoto \\ Department of Mathematical Science, University of Tokyo, Hongo, Tokyo 113, Japan
}

Received January 11, 1990; in revised form July 23, 1992

\begin{abstract}
We give the correspondence between instantons on $S^{4}$ and some representations of an associative algebra. For the given structure group, we get simultaneous imbeddings to $\mathbb{C}^{\infty}$ (the inductive limit) of the moduli spaces for instantons on $S^{4}$ of all instanton numbers.
\end{abstract}

In this note we show that instantons on $S^{4}$ can be identified with some representations of an associative algebra.

Let $A$ be the free algebra over $\mathbb{C}$ generated by two elements $q, p$. We define a new multiplication $*$ in $A$ as follows:

$$
f_{1} * f_{2}=f_{1}(p q-q p) f_{2}, \quad f_{1}, f_{2} \in A
$$

Then $(A, *)$ is an associative algebra (with no unit), which is an extention of the Weyl algebra $\mathbb{C}\left[q, \frac{d}{d q}\right]$. We consider finite dimensional representations of $(A, *)$. Let $W$ be the complex vector space of dimension $l$, and $h$ be a linear map from $A$ to End $W$. Then $h$ induces a linear map $\tilde{h}: A \otimes W \rightarrow A^{*} \otimes W$ defined by

$$
\left\langle\tilde{h}\left(f_{1} \otimes w\right), f_{2}\right\rangle=h\left(f_{2} f_{1}\right) w, f_{1}, f_{2} \in A, w \in W .
$$

We denote by $H(l, k)$ the set of all algebra homomorphisms $h:(A, *) \rightarrow$ End $W$ such that the rank of $\tilde{h}$ is $k$. If $h$ is an algebra homomorphism from $(A, *)$ to End $W$, then

$$
h\left(f_{1}(p q-q p) f_{2}\right)=h\left(f_{1}\right) h\left(f_{2}\right),
$$

so the linear map $h$ is determined by $h\left(q^{j} p^{i}\right), i, j \geqq 0$.

Let $P$ be the principal $S U(l)$ bundle over $S^{4}=\mathbb{R}^{4} \cup \infty$ with $c_{2}=k$, and $\tilde{M}(S U(l), k)$ be the framed moduli space for anti-self-dual (ASD) connections on $P$ : $\{$ ASD connections on $P\} / \mathscr{G}_{\infty}$, where $\mathscr{G}_{\infty}$ stands for the group of all gauge transformations on $P$ fixing the points in the fiber over $\infty . \tilde{M}(S U(l), k)$ is a $4 k l$ dimensional smooth manifold [1]. 
Our main result is the following:

Theorem 1. The framed moduli space $\tilde{M}(S U(l), k)$ is diffeomorphic to $H(l, k)$.

This gives an algebraic affine imbedding of $\bigsqcup_{k} \tilde{M}(S U(l), k)$ explicitly. We use Donaldson's theorem [1] to prove Theorem 1. In Sect. 1, we give a criterion in terms of linear algebra for the stability condition in Donaldson's theorem (Proposition 2). We prove Theorem 1 in Sect. 2.

\section{Some Remarks on a Theorem of Donaldson}

Let $X=\operatorname{Mat}(k, k ; \mathbb{C}) \times \operatorname{Mat}(k, k ; \mathbb{C}) \times \operatorname{Mat}(l, k ; \mathbb{C}) \times \operatorname{Mat}(k, l ; \mathbb{C})$. We define the action of $G=G L(k, \mathbb{C})$ on $X$ as follows:

$$
p \cdot\left(\alpha_{1}, \alpha_{2}, a, b\right)=\left(p \alpha_{1} p^{-1}, p \alpha_{2} p^{-1}, a p^{-1}, p b\right)
$$

for $p \in G,\left(\alpha_{1}, \alpha_{2}, a, b\right) \in X$. We call a point $x$ in $X$ stable when the map $G \ni p \mapsto p \cdot x \in X$ is proper. We denote by $X^{s}$ the set of all stable points in $X$. Let

$$
\begin{aligned}
\omega\left(\alpha_{1}, \alpha_{2}, a, b\right) & =\operatorname{tr}\left(d \alpha_{1} \wedge d \alpha_{2}+d b \wedge d a\right), \\
\mu & =\alpha_{1} \alpha_{2}-\alpha_{2} \alpha_{1}+b a .
\end{aligned}
$$

The 2-form $\omega$ is a holomorphic symplectic structure on $X$. We can show by easy computation that

$$
\begin{aligned}
\omega\left(p \alpha_{1} p^{-1}, p \alpha_{2} p^{-1}, a p^{-1}, p b\right)= & \omega\left(\alpha_{1}, \alpha_{2}, a, b\right)+\operatorname{tr}\left(p^{-1} d p \wedge d \mu\right) \\
& +\operatorname{tr}\left(p^{-1} d p \wedge p^{-1} d p \cdot \mu\right) .
\end{aligned}
$$

This means that $G$-action on $X$ preserves $\omega$ and that $\mu$ is the holomorphic moment map. (This is suggested to the author by $\mathrm{H}$. Nakajima from the viewpoint of hyperkähler structure.)

Theorem (Donaldson [1]). The framed moduli space $\tilde{M}(S U(l), k)$ is diffeomorphic to $G \backslash \mu^{-1}(0) \cap X^{s}$.

So we deduce from geometric invariant theory [4] that $\tilde{M}(S U(l), k)$ is a nonsingular quasiaffine algebraic variety. Theorem 1 gives an affine imbedding of $\tilde{M}(S U(l), k)$ explicitly and simultaneously for all $k$.

Donaldson gave a criterion for the stability in $\mu^{-1}(0)$ :

Proposition (Donaldson [1]). The point $x=\left(\alpha_{1}, \alpha_{2}, a, b\right) \in \mu^{-1}(0)$ is stable if and only if

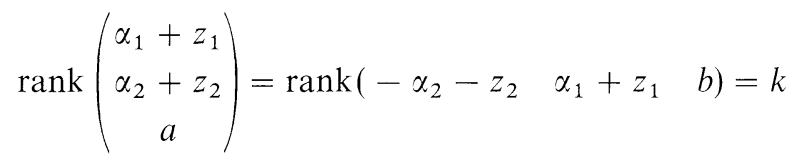

for all $z_{1}, z_{2} \in \mathbb{C}$.

Here we seek a criterion for the stability in $X$.

Proposition 2. For any point $x=\left(\alpha_{1}, \alpha_{2}, a, b\right) \in \mu^{-1}(0)$, the condition (1) is equivalent to the following:

$$
\bigcap_{f \in A} \operatorname{Ker} a f\left(\alpha_{1}, \alpha_{2}\right)=0, \quad \sum_{f \in A} \operatorname{Im} f\left(\alpha_{1}, \alpha_{2}\right) b=\mathbb{C}^{k}
$$


Proof. It is clear that (2) implies (1). Suppose that the vector space generated by the row vectors of af $\left(\alpha_{1}, \alpha_{2}\right) b(f \in A)$ is

$$
\left\{(x, 0) \in \mathbb{C}^{j} \oplus \mathbb{C}^{k-j}\right\}, \quad j<k .
$$

According to the splitting $\mathbb{C}^{k}=\mathbb{C}^{j} \oplus \mathbb{C}^{k-j}$ we set

$$
\alpha_{1}=\left(\begin{array}{ll}
\alpha_{11} & \alpha_{12} \\
\alpha_{13} & \alpha_{14}
\end{array}\right), \quad \alpha_{2}=\left(\begin{array}{ll}
\alpha_{21} & \alpha_{22} \\
\alpha_{23} & \alpha_{24}
\end{array}\right), \quad a=\left(\begin{array}{ll}
a^{\prime} & 0
\end{array}\right) \text {. }
$$

Then for any $f \in A$,

$$
\begin{gathered}
a f\left(\alpha_{1}, \alpha_{2}\right)=\left(a^{\prime} f\left(\alpha_{11}, \alpha_{21}\right) \quad 0\right), \\
a^{\prime} f\left(\alpha_{11}, \alpha_{21}\right) \alpha_{12}=0, \\
a^{\prime} f\left(\alpha_{11}, \alpha_{21}\right) \alpha_{22}=0 .
\end{gathered}
$$

So we have $\alpha_{12}=\alpha_{22}=0$, then

$$
\alpha_{1} \alpha_{2}-\alpha_{2} \alpha_{1}+b a=\left(\begin{array}{lc}
* & 0 \\
* & \alpha_{14} \alpha_{24}-\alpha_{24} \alpha_{14}
\end{array}\right) .
$$

This implies $\alpha_{14} \alpha_{24}=\alpha_{24} \alpha_{14}$. Thus there exists a nonzero common eigenvector $x^{\prime} \in \mathbb{C}^{k-j}$ of $\alpha_{14}, \alpha_{24}$. Then $\left(\begin{array}{c}0 \\ x^{\prime}\end{array}\right)$ is a nonzero common eigenvector of $\alpha_{1}, \alpha_{2}$ contained in Ker $a$. That contradicts with (1). It goes similarly in the case that the column vectors of $f\left(\alpha_{1}, \alpha_{2}\right) b(f \in A)$ does not generate whole $\mathbb{C}^{k}$.

\section{The Proof of Theorem 1}

First we give the map $\varphi$ from $\tilde{M}(S U(l), k)$ to $H(l, k)$. Let

$$
h(f)=\varphi\left(\alpha_{1}, \alpha_{2}, a, b\right)(f)=a f\left(\alpha_{1}, \alpha_{2}\right) b
$$

for $\left(\alpha_{1}, \alpha_{2}, a, b\right) \in \mu^{-1}(0) \cap X^{s} . \varphi$ is $G$-invariant. Since $\mu\left(\alpha_{1}, \alpha_{2}, a, b\right)=0$,

$$
\begin{aligned}
h\left(f_{1} * f_{2}\right) & =h\left(f_{1}(p q-q p) f_{2}\right) \\
& =a f_{1}\left(\alpha_{1}, \alpha_{2}\right)\left(\alpha_{2} \alpha_{1}-\alpha_{1} \alpha_{2}\right) f_{2}\left(\alpha_{1}, \alpha_{2}\right) b \\
& =a f_{1}\left(\alpha_{1}, \alpha_{2}\right) b a f_{2}\left(\alpha_{1}, \alpha_{2}\right) b \\
& =h\left(f_{1}\right) h\left(f_{2}\right) .
\end{aligned}
$$

We give $i: \mathbb{C}^{k} \rightarrow A^{*} \otimes \mathbb{C}^{l}, j: A \otimes \mathbb{C}^{l} \rightarrow \mathbb{C}^{k}$ by

$$
\begin{aligned}
& \langle i(v), f\rangle=a f\left(\alpha_{1}, \alpha_{2}\right) v, \\
& j(f \otimes w)=f\left(\alpha_{1}, \alpha_{2}\right) b w
\end{aligned}
$$

for $f \in A, v \in V, w \in W$. Then we have $\tilde{h}=i \circ j$. Proposition 2 implies that $i$ is injective and that $j$ is surjective, so rank $\tilde{h}=k$. Therefore $h \in H(l, k)$.

On the other hand, the inverse $\psi: H(l, k) \rightarrow \tilde{M}(S U(l), k)$ is defined as follows. For $h^{\prime} \in H(l, k)$, we set $V=\operatorname{Im} \tilde{h} \cong \mathbb{C}^{k}$. Let

$$
\begin{array}{ll}
\bar{h}^{\prime}=i^{\prime} \circ j^{\prime}, & i^{\prime}: V \rightarrow A^{*} \otimes W, \\
& j^{\prime}: A \otimes W \rightarrow V .
\end{array}
$$


For $f \in A$ we define $\langle f|\in \operatorname{Hom}(V, W)| f,\rangle \in \operatorname{Hom}(W, V)$ by

$$
\begin{gathered}
\langle f|(v)=\left\langle i^{\prime}(v), f\right\rangle, \quad v \in V, \\
|f\rangle(w)=j^{\prime}(f \otimes w), \quad w \in W .
\end{gathered}
$$

We set $a^{\prime}=\left\langle 1\left|, b^{\prime}=\right| 1\right\rangle$. The multiplications by $q, p$ in $A$ induce linear maps $\alpha_{1}^{\prime}, \alpha_{2}^{\prime} \in$ End $V$ respectively:

$$
\alpha_{1}^{\prime}|f\rangle=|q f\rangle, \quad \alpha_{2}^{\prime}|f\rangle=|p f\rangle
$$

for $f \in A$. If $|f\rangle=0$, then $h\left(f^{\prime} f\right)=0$ for all $f^{\prime} \in A$. So $\alpha_{1}^{\prime}, \alpha_{2}^{\prime} \in$ End $V$ are welldefined. We get

$$
\psi\left(h^{\prime}\right)=\left(\alpha_{1}^{\prime}, \alpha_{2}^{\prime}, a^{\prime}, b^{\prime}\right) \in X
$$

by fixing the basis of $V, W$. Since

$$
\begin{gathered}
\bigcap_{f \in A} \operatorname{Ker} a^{\prime} f\left(\alpha_{1}^{\prime}, \alpha_{2}^{\prime}\right)=\bigcap_{f \in A} \operatorname{Ker}\langle f|=0, \\
\sum_{f \in A} \operatorname{Im} f\left(\alpha_{1}^{\prime}, \alpha_{2}^{\prime}\right) b^{\prime}=\sum_{f \in A} \operatorname{Im}|f\rangle=V,
\end{gathered}
$$

we deduce from Proposition 2 that $\psi\left(h^{\prime}\right)$ is stable. Since $h^{\prime}:(A, *) \rightarrow$ End $W$ is an algebra homomorphism, we have

$$
\begin{aligned}
\left\langle f_{1}\left|\alpha_{1}^{\prime} \alpha_{2}^{\prime}-\alpha_{2}^{\prime} \alpha_{1}^{\prime}+b^{\prime} a^{\prime}\right| f_{2}\right\rangle & =h^{\prime}\left(f_{1}(q p-p q) f_{2}\right)+\left\langle f_{1} \mid 1\right\rangle\left\langle 1 \mid f_{2}\right\rangle \\
& =-h^{\prime}\left(f_{1} * f_{2}\right)+h^{\prime}\left(f_{1}\right) h^{\prime}\left(f_{2}\right) \\
& =0 .
\end{aligned}
$$

Therefore $\psi\left(h^{\prime}\right) \in G \backslash \mu^{-1}(0) \cap X^{s}$.

$$
\begin{aligned}
& \text { If }\left(\alpha_{1}^{\prime}, \alpha_{2}^{\prime}, a^{\prime}, b^{\prime}\right)=\psi\left(h^{\prime}\right), \\
& \qquad \begin{aligned}
a^{\prime} f\left(\alpha_{1}^{\prime}, \alpha_{2}^{\prime}\right) b^{\prime} & =\left\langle 1\left|f\left(\alpha_{1}^{\prime}, \alpha_{2}^{\prime}\right)\right| 1\right\rangle \\
& =\langle 1 \mid f\rangle \\
& =h^{\prime}(f) .
\end{aligned}
\end{aligned}
$$

Hence $\varphi \circ \psi\left(h^{\prime}\right)=h^{\prime}$.

If $h^{\prime}=\varphi\left(\alpha_{1}, \alpha_{2}, a, b\right)$, we can take $i^{\prime}=i, j^{\prime}=j$ by the stability. Then

$$
\left\langle f\left|=a f\left(\alpha_{1}, \alpha_{2}\right), \quad\right| f\right\rangle=f\left(\alpha_{1}, \alpha_{2}\right) b .
$$

This implies that

$$
\begin{gathered}
\langle 1|=a, \quad| 1\rangle=b, \\
|q f\rangle=\alpha_{1} f\left(\alpha_{1}, \alpha_{2}\right) b=\alpha_{1}|f\rangle, \\
|p f\rangle=\alpha_{2} f\left(\alpha_{1}, \alpha_{2}\right) b=\alpha_{2}|f\rangle .
\end{gathered}
$$

Hence $\psi \circ \varphi=\mathrm{id}$.

Acknowledgement. The author would like to express his thanks to Professor A. Hattori for much help and encouragement. 


\section{References}

1. Donaldson, S.K.: Instantons and geometric invariant theory. Commun. Math. Phys. 93 453-460 (1984)

2. Furuta, M., Hashimoto, Y.: Invariant instantons on $\mathrm{S}^{4}$. J. Fac. Sci. Univ. Tokyo Sect. IA Math. 37 (1990)

3. Hashimoto, Y.: Group actions on the moduli spaces for instantons over $\mathrm{S}^{4}$. Master thesis (in Japanese) 1987

4. Mumford, D., Fogarty, J.: Geometric Invariant Theory, 2nd Edition. Berlin Heidelberg New York: Springer 1982

Communicated by A. Jaffe 
